Trauma Berufskrankh $2012 \cdot$ 14[Suppl 3]:372-378 DOI 10.1007/s10039-012-1839-z

Online publiziert: 22. März 2012

(c) Springer-Verlag 2012

L.M. Wessel

Kinderchirurgische Klinik, Universitätsmedizin Mannheim

\title{
Verletzungen am proximalen Radius
}

\section{Therapieprinzipien, Komplikationen, Fallstricke und Korrekturoperationen}

teten Zug am Ellenbogen, den das Kleinkind muskulär nicht stabilisieren kann, zur Subluxation des Köpfchens [25, 29]. Es wird davon ausgegangen, dass sich der Radiuskopf unterhalb des Ringbandes verfängt und in Subluxationsstellung verharrt [37]. Zeichen dafür sind die sowohl radiologisch als auch sonographisch nicht korrekte Beziehung des Radiuskopfs zum Capitulum humeri [38]: Es liegt zwar keine echte Luxation vor, aber die Verlängerung des Radiushalses weist nicht auf das Zentrum des Capitulum humeri (- Abb. 1).

\section{Ursache}

In etwa 10\% der Fälle wird ein Sturz auf den Arm oder die Hand als Auslöser angegeben. Obwohl dies einen möglichen Unfallhergang darstellt, muss der unerwartete Zug am ausgestreckten Arm als Hauptursache angesehen werden [33, 37]. Dieser Unfallmechanismus sollte ohne Vorwurf auf verständnisvolle Weise von den Eltern als mögliche Erklärung erfragt werden. Er wird aus Scham oder Angst vor dem Vorwurf der Kindesmisshandlung häufig verschwiegen [38].

\section{Diagnostik}

Die Diagnose wird stets klinisch gestellt. Die typische Anamnese mit dem klinischen Bild einer Pseudolähmung im Ellenbogen bzw. Unterarmbereich lenkt zur richtigen Diagnose. Typischerweise ist das Ellenbogengelenk leicht gebeugt, und der Unterarm wird vom Kind selbst in Pronation gehalten. Das Kind bewegt den Arm und v. a. das Ellenbogengelenk nicht. Eine Schwellung oder Deformierung der oberen Extremität, insbesondere des Ellenbogengelenks, liegen nie vor Periphere Durchblutung und Sensibilität sind stets ungestört. In solchen Fällen ist eine weitere Diagnostik nie sinnvoll.

Besteht Zweifel an der Diagnose bzw. ist der Unfallhergang atypisch (Sturzanamnese), kann die Diagnose mit Hilfe der Sonographie elegant und ohne Strahlenbelastung bestätigt bzw. ausgeschlossen werden.

Beim klinischen Verdacht einer knöchernen Verletzung sollte eine konventionelle Radiologie des Ellenbogens in 2 Ebenen erfolgen, um Frakturen des distalen Humerus oder proximalen Unterarms (sehr selten) auszuschließen. Eine Vergleichsaufnahme der nicht verletzten Seite muss grundsätzlich unterbleiben, da diese nie zielführend und aus strahlenhygienischen Gründen abzulehnen ist.

Wird die Diagnose aufgrund der Klinik gestellt, ist das Risiko, eine Fraktur zu übersehen, vernachlässigbar gering (relatives Risiko 1,2, 95\%-Konfidenzinterall $0,8-1,5 ;[24])$.

\section{Therapie}

Wurde die Diagnose bereits klinisch gestellt, kann man - nach Aufklärung der Eltern - sofort mit der Behandlung, der 

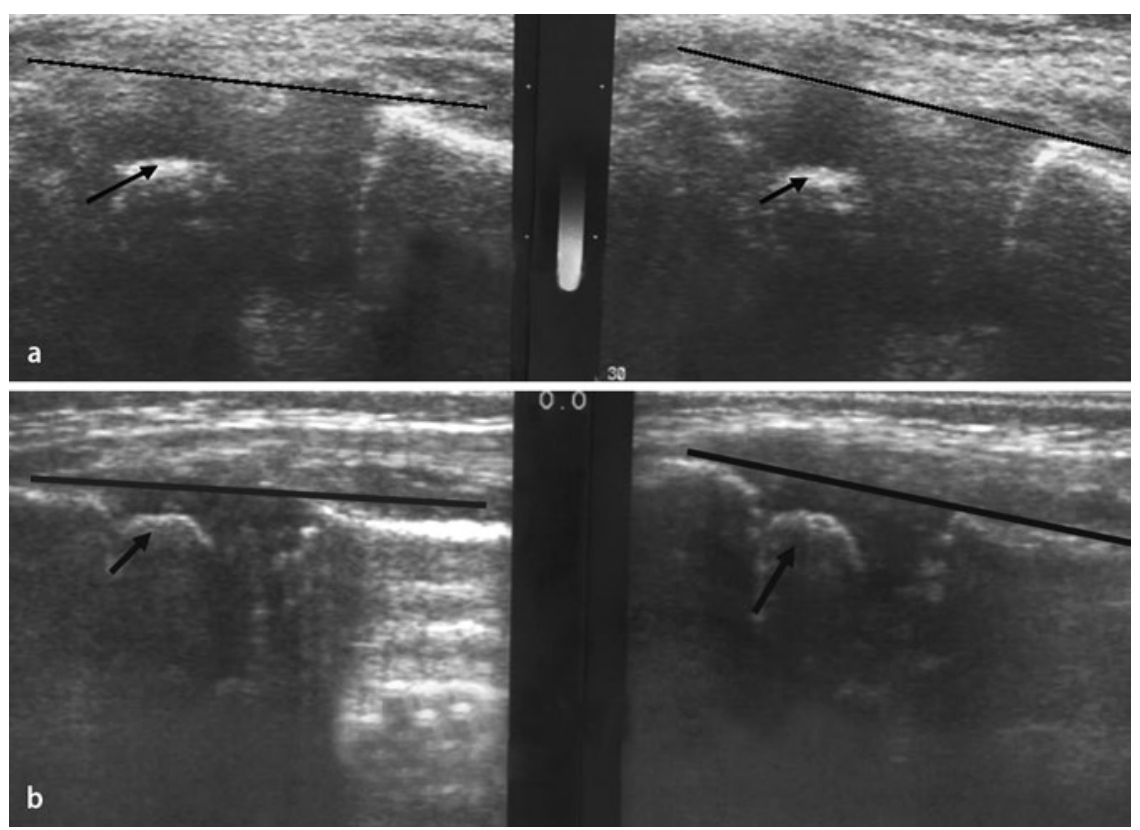

Abb. 1 \ Sonographie des Ellenbogengelenks nach Zug am Arm mit klinischem Vorliegen einer Subluxation des Radiuskopfs, schwarze Pfeile Kern des Capitulums, weiter proximal distale Metaphyse des Humerus, schwarze Linie Ebene des Radiushalses, vor Reposition (a) deutlich größere Distanz als nach Reposition (b)

Reposition der Subluxation, beginnen. Am einfachsten gelingt diese durch eine Hyperpronation im Ellenbogengelenk, indem man den Unterarm in der vorhandenen Stellung forciert proniert. Durch die Reposition des Radiusköpfchens kommt es $\mathrm{zu}$ einem deutlichen Klickgeräusch $[23,24]$. Sie ist für einen kurzen Moment schmerzhaft, und in der Regel schreit das Kind kurz auf. Dieses Aufschreien beruht auf einer Mischung aus Angst vor jeglicher Manipulation, die verständlicherweise bereits schmerzhaft ist, und einem kurzen Schmerz bei der Reposition selbst. Kurz darauf beruhigen sich die Kinder und setzen den Arm und Ellenbogen wieder uneingeschränkt ein. Dies kann leicht überprüft werden, indem man das Kind ein Spielzeug oder ein Bonbon mit dem betroffenen Arm greifen lässt. Ist die Greiffunktion wieder hergestellt, beweist das die erfolgreiche Behandlung. Eine Ruhigstellung im Gips- oder Stützverband ist nicht notwendig.

Eine alternative Reposition wird durch Supination des Unterarms mit voller Streckung und anschließender Beugung erreicht [6].

Vergleicht man beide Methoden, weist die Hyperpronation eine Erfolgsrate von etwa $97 \%$ gegenüber $85 \%$ bei der Supi- nationsmethode auf. Zudem ist Letztere deutlich schmerzhafter und aufwendiger. Gelingt die Reposition nicht sicher oder hält das Kind den Unterarm weiterhin in Schutzhaltung, führt eine kurzzeitige $\mathrm{Ru}$ higstellung auf einer Oberarmgipsschiene von maximal 1 Woche stets zur problemlosen Heilung.

\section{Fallstricke}

Komplikationen und Fallstricke der Behandlung sind die grundsätzliche Anfertigung von Röntgenaufnahmen sowie die fehlende Aufklärung über die schmerzhafte Reposition. Durch die einfache Handhabung und den sofortigen Erfolg ist diese Maßnahme ohne Narkose oder Schmerzmittel absolut vertretbar. Es ist wichtig, die Eltern über die wahre Ursache der Subluxation und deren Dauer (bis zum 4. Lebensjahr) aufzuklären.

Sollte es zum Rezidiv kommen, hat sich eine kurzfristige Ruhigstellung (bis maximal 10 Tage) mit Hilfe einer Oberarmgipsschiene absolut bewährt. Dadurch kann weiteren Rezidiven vorgebeugt werden, wie in einer randomisierten Studie nachgewiesen werden konnte [39]. Die Aufklärung der Erziehungsberechtigten ist die wirksamste Prophyla- xe. Prinzipiell kann die Heilung auf Dauer nicht verhindert werden. Eine operative Revision des Ellenbogengelenks wäre ein Kunstfehler. Spätfolgen wurden in der Literatur noch nie beschrieben. Somit entfällt die Empfehlung zu einer Korrekturoperation.

\section{Frakturen des Radiuskopfs bzw. -halses}

\section{Definition, Ursache und Epidemiologie}

Frakturen des Radiuskopfs bzw. -halses sind selten und in höchstens $1 \%$ aller Extremitätenverletzungen anzutreffen. Auslösend sind der Sturz mit Varuskomponente auf den ausgestreckten Arm mit proniertem Unterarm bzw. mit Valguskomponente beim Sturz auf den supinierten Unterarm. Durch diesen Unfallmechanismus kommt es in 70\% der Fälle zu metaphysären Frakturen im Halsbereich und in etwa 30\% zu Fugenlösungen (Harris-Salter-Fraktur-Typ I und II; [19]). Meißelfrakturen im Sinne einer Harris-Salter-Fraktur-Typ IV sind selten und kommen ausschließlich im Jugendalter vor $[8,22]$. Es handelt sich dann um echte Wachstumsfugenfrakturen (extrem selten) oder aber um Übergangsfrakturen (etwas häufiger, analog den Übergangsfrakturen der distalen Tibia).

\section{Problematik der proximalen Radiusfrakturen}

Sie erklärt sich durch die delikate Durchblutung des Kopfs. Verplumpungen des Radiusköpfchens aufgrund von Kopfumbaustörungen, aseptischen Nekrosen und periartikulären Verkalkungen sind die häufigsten Komplikationen $[8,28]$. Die besten Langzeitergebnisse werden nach konservativer Therapie erzielt [11, 17, 42].

\section{Therapie}

\section{Prinzipien}

Sie beinhalten zunächst eine Abklärung der Wachstumsprognose. Eine wie auch immer geartete Korrektur ist nur möglich, so lange die Fugen noch offen sind, was nicht vom Alter des Kindes bestimmt wird [20]: Die ellenbogennahen Wachs- 
tumsfugen verschließen sich als eine der ersten im Körper, bei Mädchen früher als bei Jungen. Es gibt außerdem ein NordSüd-Gefälle, nordische Kinder wachsen länger als Kinder aus dem Mittelmeerraum. So ist es gut möglich, dass bei 10-jährigen Mädchen die Fugen am Ellenbogengelenk bereits verschlossen sind, obwohl die meisten anderen Fugen noch weiteres Wachstumspotenzial besitzen.

Obwohl das Längenwachstum der oberen Extremität an den Fugen um das Ellenbogengelenk insgesamt nur $20 \%$ beträgt, ist die Korrekturpotenz gerade der proximalen Radiusfrakturen bis ungefähr zum 10. Lebensjahr sehr hoch. Allerdings gilt dies nur für Achsabweichungen; Korrekturen bis $45^{\circ}$ remodellieren sich im Laufe des restlichen Wachstums zuverlässig, wie beeindruckende Fallbeispiele im Lehrbuch von von Laer et al. [20] zeigen. Dies gilt nicht für Ad-latus-Verschiebungen. Der Radiuskopf ist dann nicht mehr exakt auf das Capitulum hin zentriert, sodass die Umwendbewegungen im Ellenbogengelenk erheblich eingeschränkt sind [34]. Jenseits des 10. Lebensjahrs werden Abkippungen nur noch begrenzt (höchstens bis $10^{\circ}$ ) spontan korrigiert. In dieser Altersgruppe kann somit keine ausgeprägte Fehlstellung toleriert werden.

\section{Vorgehensweise}

Die Reposition sollte auf jeden Fall sehr schonend erfolgen. Eine traumatische Reposition kann die Durchblutung komplett zerstören, sodass später eine vollständige Nekrose des Radiuskopfs mit Umbaustörung und konsekutiver Funktionseinschränkung im Ellenbogengelenk resultiert. Die schlimmste Form der Funktionseinschränkung ist die proximale radioulnare Synostose, die nach erheblicher Dislokation, offener Reposition und konsekutiver aseptischer Nekrose auftreten kann [5]. Eine schonende Methode ist die Technik nach Israeli. Hierbei wird in Narkose am Unterarm gezogen und gleichzeitig sanfter Druck auf den Radiuskopf ausgeübt, während der Unterarm passiv im Sinne der Umwendbewegung in Pronation gedreht wird [9].

Mehr als 2 Repositionsversuche sind nicht sinnvoll. Zum einen gelingt die Reposition selten bei einer weiteren Manipulation und zum anderen kann die gewalt-

Trauma Berufskrankh 2012 - 14[Suppl 3]:372-378 DOI 10.1007/s10039-012-1839-z

(c) Springer-Verlag 2012

\section{L.M. Wessel \\ Verletzungen am proximalen Radius. Therapieprinzipien, Komplikationen, Fallstricke und Korrekturoperationen}

\section{Zusammenfassung}

Knöcherne Verletzungen im Ellenbogengelenkbereich im Kindesalter sind selten, aber gefürchtet, da sie häufig mit Komplikationen einhergehen und eine schlechte Spontankorrektur aufweisen. Gegenüber Erwachsenen bestehen erhebliche Unterschiede in Verletzungsmuster, Therapie und Prognose. Vergleichsaufnahmen der nicht verletzen Gegenseite sind grundsätzlich abzulehnen. Die Subluxation des Radiuskopfs (Chassaignac-Verletzung) tritt nur im Kleinkindesalter auf und sollte stets klinisch diagnostiziert werden. Sie wird durch Hyperpronation des Unterarms behandelt. Frakturen des proximalen Radius (Radiuskopffraktur) im Kindesalter kommen fast ausschließlich als Radiushalsfrakturen (metaphysär oder Fugenlösungen) vor. Wegen der Beeinträchti- gung der Durchblutung sind Kopfumbaustörungen häufig. Die besten Ergebnisse werden mit der konservativen Therapie erzielt. Die Monteggia-Fraktur hat im Kindesalter eine bessere Prognose als bei Erwachsenen, vorausgesetzt die Radiuskopfluxation wird richtig diagnostiziert. Eine konservative Behandlung ist in etwa 50\% der Fälle möglich. Korrekturoperationen nach übersehener Verletzung sind mittels Fixateur-externe-Einsatz möglich, zeigen aber schlechtere Ergebnisse als nach regelrechter Erstbehandlung.

\section{Schlüsselwörter}

Radiusverletzungen · Kindesalter · Radiuskopf- und -halsverletzungen . Monteggia-Frakturen $\cdot$ Korrekturoperationen

\section{Injuries of the proximal radius. Current treatment options, complications, pitfalls and reconstructive procedures}

\begin{abstract}
Osseous lesions and injuries around the elbow joint in children are feared lesions since the turn of the nineteenth century. Complications are often and blunders in treatment have often been published. Skeletal healing and remodelling is modest. Furthermore there are other characteristics than in adult lesions related to the pattern of lesions, diagnostics and treatment. Comparative $x$-rays of the non-injured side are strictly contraindicated. Radial head subluxation is a typical lesion of toddlers and young children and the diagnosis is made based on the history of a pulled arm and physical findings. Fractures of the radial neck are almost exclusively metaphyseal and epiphysiolysis lesions (HarrisSalter I and II). Due to blood supply disturbances a radial head overgrowth is often observed. Best therapeutic outcome is achieved
\end{abstract}

with conservative treatment. Up to the age of 9-10 years corrective potential and remodelling is excellent. Open procedures and extensive osteosynthesis are associated with major complications. The Monteggia fracture in children has a better prognosis than in adults, provided dislocation of the radial head was diagnosed and conservative treatment is possible in nearly $50 \%$ of cases. Reconstructive procedures after missed dislocations are possible but children end up with a clearly inferior result.

\section{Keywords}

Radius, injuries · Children · Radial head and neck injuries - Monteggia's fracture . Reconstructive surgical procedures same Reposition die restliche Durchblutung zerstören.

Gelingt die geschlossene Reposition nicht, sollte bei nicht tolerierbarer Fehlstellung eine intramedulläre Reposition mit Hilfe eines elastischen Marknagels (ESIN: elastisch stabiler intramedullärer Nagel) erfolgen. Mit dieser Technik wird ein etwas stärker vorgebogener ESIN auf- steigend in den proximalen Radius bis kurz vor der Fraktur vorgetrieben. Der abgekippte Kopf wird mit dem Haken am ESIN aufgespießt und in die richtige Position rotiert, sodass eine achsengerechte Stellung in 2 senkrecht aufeinander stehenden Ebenen resultiert. Der ESIN wird zur Heilung belassen. Eine frühzeitige 

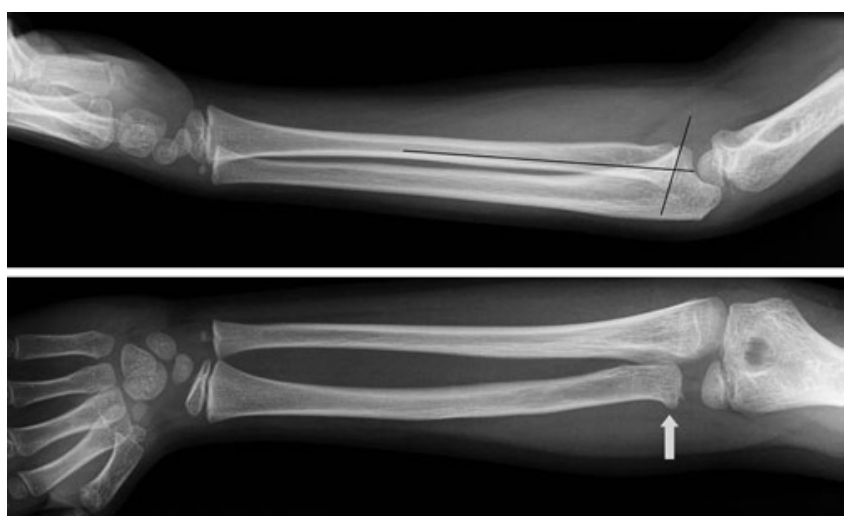

Abb. 2 \ Knapp 4-jähriger Junge nach Sturz auf den ausgestreckten Arm mit klinisch deutlicher Schwellung im Ellenbogenbereich, radial betontem Druckschmerz und nicht aktiv durchführbaren Umwendbewegungen, Röntgenbilder in Behelfstechnik: keine 2 senkrecht aufeinander stehenden Ebenen, kein Fettpolsterzeichen, Knochenkern des Radiuskopfs nicht angelegt; als Zeichen der Radiushalsfraktur mit Einstauchung: Abknickung der Gelenkebene (Linien) sowie diskrete Wulstbildung an der proximalen Radiusmetaphyse, Pfeil

funktionelle Behandlung ist möglich, eine Gipsbehandlung nicht Gewinn bringend.

Lässt sich die Fraktur geschlossen mit der Joystickmethode (ESIN) nicht reponieren, bleibt nur die offene Reposition. Hierzu wird ein radialer Zugang zum Ellenbogengelenk über dem tastbaren Radiuskopf gewählt. In der Regel muss das Ringband durchtrennt werden, damit der Kopf wieder reponiert werden kann. Die Osteosynthese erfolgt mit KirschnerDrähten. Eine aufwendige Osteosynthese mit kleinen Platten und Schrauben sollte unterbleiben, da sie unweigerlich zur aseptischen Nekrose und erheblichen Verplumpung mit Bewegungseinschränkung führt.

\section{Fallstricke}

Komplikationen hängen zum einen mit der primären Verletzung zusammen. Eine gewisse Kopfumbaustörung ist nach jeder Radiushals- oder -kopffraktur nachweisbar $[15,42]$. Durch eine erhebliche Dislokation kommt es zwangsläufig zur Durchblutungsstörung mit aseptischer Nekrose. Die meisten Schäden werden jedoch iatrogen durch eine inadäquate Behandlung verursacht. Die offene Reposition mit aufwendiger Osteosynthese führt unweigerlich zur aseptischen Nekrose und monströsen Kopfumbaustörungen mit erheblicher Beeinträchtigung der Umwendbeweglichkeit oder im schlimmsten Fall zur radioulnaren Synostose [5]. Ein Kompartmentsyndrom kann ebenfalls auftreten, ist allerdings sehr selten [32].

Fallstricke in der Diagnostik sind das Übersehen der Verletzung, v. a. wenn bei Kleinkindern der Knochenkern des Radiuskopfs noch nicht sichtbar ist. Die Fraktur ist dann an dem positiven Fettpolsterzeichen und einem diskreten Wulst an der proximalen Radiusmetaphyse erkennbar (• Abb. 2). Liegt der dislozierte Kopf hinter dem Radiusschaft, kann die Verletzung ebenfalls übersehen werden. Ein sicheres Zeichen ist die fehlende Wachstumsfuge.

In der Behandlung ist die transkondyläre Kirschner-Draht-Osteosynthese nach Witt [44] absolut obsolet. Sie führt häufig zum Drahtbruch, Infektion und/oder Kopfnekrose [20].

In der Nachbehandlung muss vor frühzeitiger intensiver Physiotherapie wegen periartikulärer Verkalkungen bzw. Verstärkung der aseptischen Nekrose gewarnt werden. Nach Gelenkmanipulationen ist eine erhebliche Myositis ossificans beschrieben [40].

Auch von der Radiuskopfresektion im Wachstumsalter muss dringend abgeraten werden, da es dann noch vor Abschluss des Wachstums zum einem erheblichen Ulnavorschub mit entsprechenden Beschwerden bis zur Arthrose im Handgelenk kommen kann.
Die operative Resektion und Korrektur einer radioulnaren Synostose kann nicht empfohlen werden, da sie in weit über $50 \%$ der Fälle rezidiviert und keine befriedigenden Ergebnisse aufweist [16, 27].

\section{Korrekturoperationen}

Für die möglichen Korrekturoperationen gilt der Grundsatz: Prävention ist besser als Korrekturversuch [14]. Langzeitergebnisse nach Radiuskopfresektion sind nicht zufrieden stellend, wobei in Einzelfällen sehr gute Resultate erzielt werden können. Operative Arthrolysen sowie Modellageoperationen am Radiuskopf wegen erheblicher Verplumpung sind selten von langfristigem Erfolg gekrönt und hängen im Wesentlichen von der Erfahrung des Operateurs und der Compliance der (meist uneinsichtigen) Jugendlichen ab. Bei persistierender Fehlstellung oder aber nach partiellem frühzeitigem Fugenschluss ist die subkapitale Umstellungsosteotomie zu erwägen. Vereinzelt wird über gute $\mathrm{Er}$ gebnisse einer Silasticprothese berichtet. Insgesamt gesehen sind die Erfolge der Korrekturoperationen eher als bescheiden einzustufen.

\section{Monteggia-Fraktur bzw. -Äquivalentfraktur}

\section{Definition, Ursache und Epidemiologie}

Die Monteggia-Fraktur ist eine Ulnafraktur in Kombination mit einer Luxation des Radiuskopfs im Ellenbogengelenk. Bei den Äquivalentverletzungen handelt es sich um eine proximale Ulna- und Radiushals- bzw. -kopffraktur. Diese Läsionen sind sowohl bei Kindern jeder Altersgruppe als auch bei Erwachsenen anzutreffen [3, 41]. Die Prognose ist im Kindesalter besser als bei Erwachsenen [31].

\section{Problematik}

Problem der Monteggia-Fraktur ist, dass die Ulnafraktur in aller Regel erkannt, die Luxation des Radiuskopfs jedoch in etwa 50\% der Fälle übersehen wird $[12,18]$. Die Abgrenzung zur kongenitalen Luxation kann ausgesprochen schwierig sein $[1,26,36]$. Hilfreich ist die Beurteilung 


\section{Verletzungen der oberen Extremitäten im Kindesalter}
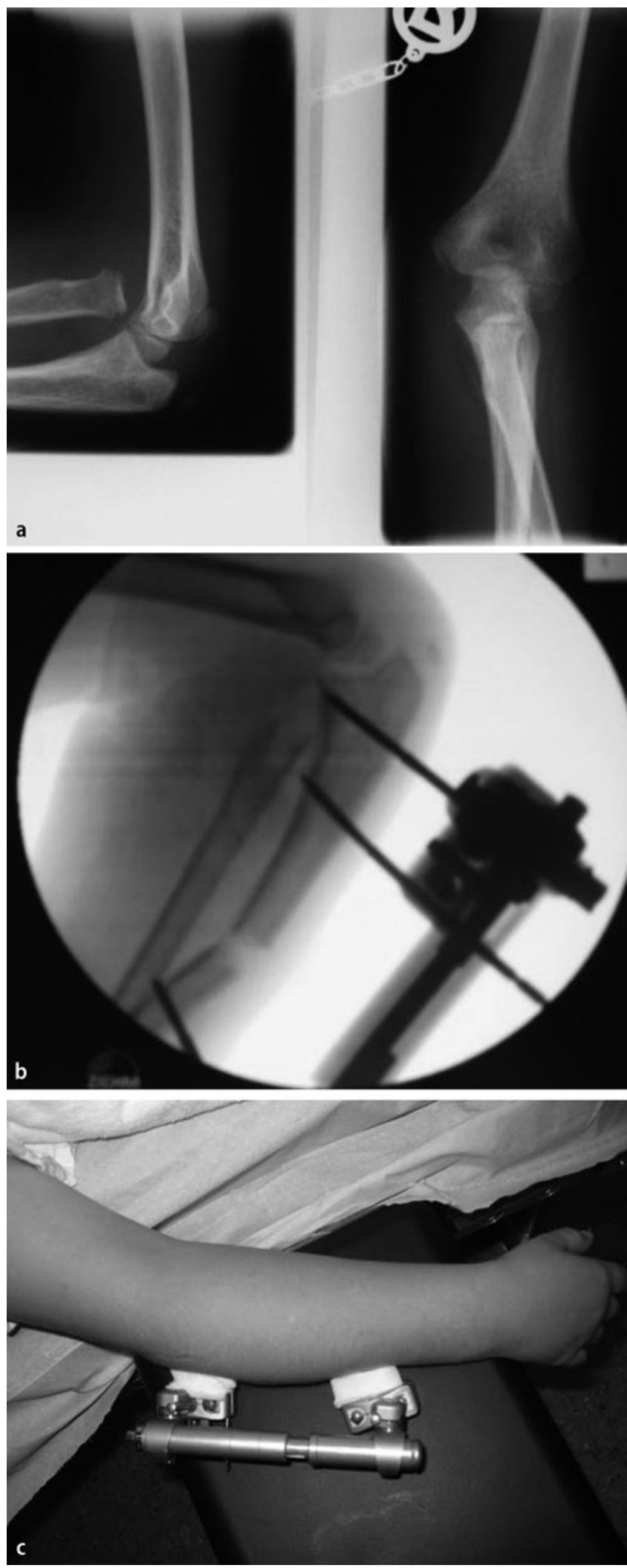

Abb. $3<6$-jähriges Mädchen nach übersehener Monteggia-Fraktur, a Röntgenbilder mit Luxation des Radiuskopfs nach ventral in der seitlichen Ebene, b Durchleuchtungsbild nach Osteotomie der Ulna und Verlängerung mit Fixateur externe unter Angulation mit regelrechter Stellung des Radiuskopfs in 2 Ebenen, c klinisches Bild des liegenden Fixateur externe der Richtung der Luxation und der Radiuskopfform. Bei der Monteggia-Verletzung ist der Radiuskopf nach ventral oder radial, nie nach dorsal luxiert, weist eine normale Form mit einer zum Capitulum passenden Pfanne auf und ist nicht abgerundet $[1,9,36]$.

Um die Luxation des Kopfs auszuschließen, muss die durch den Radiushals verlängerte Linie immer in jeder Ebene und Beugestellung des Ellenbogens auf das Zentrum des Capitulums zeigen. Ist das nicht der Fall, handelt es sich um eine Luxation und somit um eine MonteggiaFraktur.

\section{Klassifikation}

Im Erwachsenenalter werden diese Verletzungen nach Bado [3] eingeteilt. Für das Kindesalter bewährte sich die Klassifikation nach Letts et al. [21]. Die Einteilung ist für die Repositionstechnik von klinischer Relevanz [34].

\section{Therapie}

Die Therapieprinzipien sind im Kindesalter die Reposition der Ulnafraktur. Steht die Ulna wieder anatomisch, kommt es immer zur problemlosen geschlossenen Reposition des Radiuskopfs. Eine offene Reposition ist eine absolute Ausnahme. Die Art der Luxation und die der Ulnafraktur diktieren auch die Repositionstechnik:

- Ist die Ulnafraktur im Schaftbereich gelegen, ist der Kopf nach ventral luxiert. Zur Reposition werden die Länge und die Achse der Ulna wiederhergestellt und unter Zug gebeugt; dabei springt der Kopf ins Gelenk.

- Bei proximal metaphysärer Ulnafraktur luxiert der Radiuskopf nach radiodorsal. Zur Reposition wird die Ulna gestreckt und valgisiert; in Streckstellung reponiert sich der Kopf spontan.

- Ist die Ulnafraktur instabil, ist die Osteosynthese indiziert [35]. Methode der Wahl ist ein aufsteigender oder absteigender ESIN der Ulna. Nach Reposition reponiert sich der Radiuskopf spontan. 
Transkondyläre Kirschner-Draht-Osteosynthesen nach Witt [44] sollten tunlichst unterbleiben $[9,20]$.

\section{Komplikationen}

Sie entstehen, wenn die Luxation übersehen wird, was in bis zu 50\% der Fälle geschieht. Die Ulnafraktur verheilt (meistens in Fehlstellung), aber die Luxation des Radiuskopfs persistiert. Anfangs sind die Beschwerden außer einer Bewegungseinschränkung recht benigne. Mit zunehmender Dauer der Luxation (ab dem 2. posttraumatischen Jahr) treten Schmerzen und neuronale Schäden (Nn. medianus und radialis, vorwiegend sensibel) hinzu. Durch die Verplumpung des Radiuskopfs kommt es zur Bewegungseinschränkung und auf Dauer zu einer Degeneration des Radiohumeralgelenks wegen zunehmender Inkongruenz zwischen Kopf und Capitulum [43].

\section{Fallstricke}

Sie betreffen sowohl die Diagnostik als auch die Behandlung.

Zur Diagnostik muss der gesamte Unterarm mit angrenzenden Gelenken in jeweils 2 senkrecht aufeinander stehenden Ebenen geröntgt werden.

Die Luxation darf nicht übersehen werden! Ist dies dennoch der Fall, entwickeln sich die Beschwerden erst im Laufe der Jahre. Es kommt zur Valgusfehlstellung und Instabilität im Ellenbogengelenk.

Die Abgrenzung zur kongenitalen Radiuskopfluxation (meist beidseitig!) kann Schwierigkeiten bereiten.

\section{Korrekturoperationen}

Auch hier gilt: Prävention ist besser als Korrektur. Gerade einige Jahre nach der ursprünglichen Verletzung sind die Ergebnisse der Korrekturoperationen selten gut.

Im Kindesalter ist die Prognose besser als bei Erwachsenen, aber nur unter der Voraussetzung, dass die Korrektur vor Auftreten der Umbaustörung des Radiuskopfs stattfindet.

Bei der Korrekturoperation muss die Länge der Ulna wiederhergestellt werden. Bei frühzeitiger Operation gelingt dies auch mit einer Plattenosteosynthese. In der Regel müssen die Reste des Ringbandes reseziert werden; vereinzelt lässt sich dieses spalten, und der Kopf kann reponiert und das Ringband rekonstruiert werden. Die Notwendigkeit der Rekonstruktion des Ringbandes wird sehr kontrovers diskutiert; eine eindeutige Empfehlung dazu kann nicht abgegeben werden $[10,30]$.

In den letzten Jahren bewährte sich die Korrekturoperation mit Hilfe eines Fixateur externe. Die Ulna wird osteotomiert und verlängert. Findet die Korrektur bis 6 Monate nach der Erstverletzung statt, kann einzeitig vorgegangen werden. Die Ulna wird verlängert und anguliert, bis der Radiuskopf in 2 Ebenen sicher reponiert ist (• Abb. 3, [10]). Bei veralteten Fällen ist zunächst eine Ulnaverlängerung erforderlich. Ist die entsprechende Länge erreicht, werden die Ulna anguliert und der Radiuskopf repositioniert [10].

\section{Fazit für die Praxis}

Die Chassaignac-Verletzung ist eine häufige Diagnose bis zum 4. Lebensjahr. Bei eindeutiger Klinik sollte auch bei atypischer Anamnese zunächst eine Reposition in Hyperpronation versucht werden. Röntgenaufnahmen werden nur ausnahmsweise benötigt. Vergleichsaufnahmen der nicht verletzten Gegenseite sind grundsätzlich entbehrlich und als Kunstfehler einzustufen.

Radiuskopffrakturen sind im Kindesalter immer als metaphysäre Frakturen (Hals) oder als Fugenlösungen (Harris-SalterVerletzung Typ I oder II) anzutreffen. Die konservative Behandlung führt grundsätzlich zu besseren Ergebnissen als die operative Therapie. Eine gewisse Kopfumbaustörung ist aufgrund der Durchblutungssituation unvermeidbar. Physiotherapie ist in der Nachbehandlung nicht indiziert. Von Radiuskopfresektionen vor Wachstumsabschluss ist entschieden abzuraten.

Jede isolierte Ulnafraktur erfordert den Ausschluss einer Radiuskopfluxation im Sinne einer Monteggia-Fraktur. Bei stabilen Verhältnissen ist im Kindesalter die konservative Therapie die Methode der Wahl. Bei der Monteggia-Fraktur ist die
Prävention einer Korrekturoperation immer besser als die späte Korrektur. Die Radiuskopfluxation muss gesucht bzw. sicher ausgeschlossen werden!

\section{Korrespondenzadresse}

Prof. Dr. L.M. Wessel

Kinderchirurgische Klinik, Universitätsmedizin Mannheim, Theodor-Kutzer-Ufer 1-3, 68163 Mannheim Lucas.Wessel@medma.uni-heidelberg.de

Interessenkonflikt. Der korrespondierende Autor gibt an, dass kein Interessenkonflikt besteht.

The supplement containing this article is not sponsored by industry.

\section{Literatur}

1. Almquist $\mathrm{EE}$, Gordon LH, Blue Al (1969) Congenital dislocation of the head of the radius. J Bone Joint Surg Am 51:1118-1127

2. Amir D, Frankl U, Pogrund H (1990) Pulled elbow and hypermobility of joints. Clin Orthop Relat Res 257:94-99

3. Bado JL (1967) The Monteggia lesion. Clin Orthop Relat Res 50:71-86

4. Beatty JH, Kasser JR (2006) The elbow region: general concepts in the pediatric patient. In: Beatty JH, Rockwood CA, Kasser JR (Hrsg) Rockwood and Wilkins' fractures in children. Lippincott Williams \& Wilkins, Philadelphia, S 529-542

5. Benz G, Roth H (1985) Zur Problematik der Radiusköpfchenfraktur beim Kind. Z Kinderchir 40:289293

6. Choung W, Heinrich SD (1995) Acute annular ligament interposition into the radiocapitellar joint in children (nursemaid's elbow). J Pediatr Orthop 15:454-456

7. Costignan PG (1952) Subluxation of the annular ligament at the proximal radio-ulnar joint. Alberta Med Bull 17:7-9

8. D'Souza S, Vaishya R, Klenerman L (1993) Management of radial neck fractures in children: a retrospective analysis of one hundred patients. J Pediatr Orthop 13:232-238

9. Erickson M, Frick S (2009) Fractures of the proximal radius and ulna. In: Beatty JH, Rockwood CA, Kasser JR (Hrsg) Rockwood and Wilkins' fractures in children. Lippincott Williams \& Wilkins, Philadelphia, S 405-444

10. Exner J (2001) Missed chronic anterior Monteggia lesion. Closed reduction by gradual lengthening and angulation of the ulna. J Bone Joint Surg Br 83:547-550

11. Fowles JV, Kassab MT (1986) Observations concerning radial neck fractures in children. J Pediatr Orthop 6:51-57

12. Gleeson AP, Beattie TF (1994) Monteggia fracturedislocation in children. J Accid Emerg Med 11:192194

13. Griffin ME (1955) Subluxation of the head of the radius in young children. Pediatrics 15:103-106

14. Günther P, Wessel LM (2002) Korrektureingriffe nach ellenbogennahen Frakturen. Unfallchirurg 105(5): 478-482 
15. Hässle M, Mellerowicz H (1991) Frakturen des proximalen Radius im Wachstumsalter. Unfallchirurg 17:24-33

16. Herndon JH, Williams JJ, Weidman CD (1990) Radial growth and function of the forearm after excision of the radial head. A study of growing macaque monkeys. J Bone Joint Surg Am 72:736-741

17. Jones ER, Esah M (1971) Displaced fractures of the neck of the radius in children. J Bone Joint Surg Br 53:429-439

18. Kay RM, Skaggs DL (1998) The pediatric Monteggia fracture. Am J Orthop 27:606-609

19. Landin LA (1983) Fracture patterns in children. Analysis of 8,682 fractures with special reference to incidence, etiology and secular changes in a Swedish urban population 1950-1979. Acta Paediatr Scand Suppl 202:1-109

20. Laer L von, Kraus R, Linhart W (2007) Verletzungen im Bereich des Ellbogens. In: Laer L von, Kraus R, Linhart W (Hrsg) Frakturen und Luxationen im Kindesalter. Thieme, Stuttgart New York, S 118-191

21. Letts M, Locht R, Wiens J (1985) Monteggia fracture-dislocations in children. J Bone Joint Surg Br 67:724-727

22. Leung AG, Peterson HA (2000) Fractures of the proximal radial head and neck in children with emphasis on those that involve the articular cartilage. J Pediatr Orthop 20:7-14

23. Macias CG, Bothner J, Wiebe R (1998) A comparison of supination/flexion to hyperpronation in the reduction of radial head subluxations. Pediatrics 102:e10

24. Macias CG, Wiebe R, Bothner J (2000) History and findings associated with clinically suspected radial head subluxations. Pediatr Emerg Care 16:22-25

25. Magill HK, Aitken AP (1954) Pulled elbow. Surg Gynaecol Obstet 98:753-756

26. Miura T, Nakamura R, Horii E (1990) Congenital hand anomalies in Japan: a family study. J Hand Surg [Am] 15:439-444

27. Mikic ZD, Vukadinovic SM (1983) Late results in fractures of the radial head treated by excision. Clin Orthop Relat Res 181:220-228

28. Newman JH (1977) Displaced radial neck fractures in children. Injury 9:114-121

29. Newman J (1985) "Nursemaid's elbow" in infants six months and under. J Emerg Med 2:403-404

30. Papandrea R, Waters PM (2000) Posttraumatic reconstruction of the elbow in the paediatric patient. Clin Orthop Relat Res 370:115-126

31. Pesl T, Havranek P (2010) Monteggia lesions in the growing skeleton: principles of therapy. Acta Chir Orthop Traumatol Cech 77:32-38

32. Peters CL, Scott SM (1995) Compartment syndrome in the forearm following fractures of the radial head or neck in children. J Bone Joint Surg Am 77:1070-1074

33. Piroth P, Gharib M (1976) Die traumatische Subluxation des Radiusköpfchens (Chassaignac). Dtsch Med Wochenschr 101:1520-1523

34. Pring $M$, Wenger $D$, Rang $M$ (2005) Elbow - proximal radius and ulna. In: Rang $M$, Wenger DR, Pring ME (Hrsg) Rang's children's fractures. Lippincott Williams \& Wilkins, Philadelphia, S 120-134

35. Ring D, Waters PM (1996) Operative fixation of Monteggia fractures in children. J Bone Joint Surg $\mathrm{Br}$ 78:734-739

36. Sachar K, Mih AD (1998) Congenital radial head dislocations. Hand Clin 14:39-47

37. Sacchetti A, Ramoska EE, Glascow C (1990) Nonclassic history in children with radial head subluxations. J Emerg Med 8:151-153
38. Stans AA, Heinrich SD (2006) Dislocations of the elbow. In: Beatty JH, Rockwood CA, Kasser JR (Hrsg) Rockwood and Wilkins' fractures in children. Lippincott Williams \& Wilkins, Philadelphia, S 661702

39. Taha AM (2000) The treatment of pulled elbow: a prospective randomized study. Arch Orthop Trauma Surg 120:336-337

40. Vahvanen V, Grippenberg L (1978) Fracture of the radial neck in children. A long-term follow-up study of 43 cases. Acta Orthop Scand 49:32-38

41. Vinz H (1989) Die Monteggiafraktur im Kindesalter. Beitr Orthop Traumatol 36:153-168

42. Vocke AK, Laer L von (1989) Displaced fractures of the radial neck in children: long-term results and prognosis of conservative treatment. J Pediatr Orthop B 7:217-222

43. Wilkins KE (2002) Changes in the management of Monteggia fractures. J Pediatr Orthop 22:548-554

44. Witt AN (1965) Die transartikuläre Fixation bei Frakturen und Luxationen im Bereich des Humeroradialgelenks. In: Maurer G (Hrsg) Chirurg im Fortschritt. Enke, Stuttgart, S 45 